\title{
Toward Less-Invasive Management of Early-Stage Breast Cancer
}

Presented by Amy Cyr, MD

\begin{abstract}
Patients with early-stage breast cancer today are benefitting from a growing trend toward less-invasive disease staging and management. This is occurring as a result of molecular profiling to refine treatment, surgical approaches that improve cosmesis, radiotherapy approaches that are more convenient and less likely to produce toxicity, and the discontinuation of routine axillary dissection. Less-aggressive treatments yield better quality of life, which is very important in a malignancy with excellent long-term outcomes. (J Natl Compr Canc Netw 2015;13:646-648)
\end{abstract}

The management of early-stage breast cancer is becoming less and less invasive, according to Amy Cyr, MD, Assistant Professor of Surgery at Washington University School of Medicine and an oncologic surgeon at the Siteman Cancer Center at Barnes-Jewish Hospital.

The trend toward less-invasive assessment and treatment touches on all aspects of care: staging, surgery, radiotherapy, and systemic therapy, Dr. Cyr said at the NCCN 20th Annual Conference.

"Patients often come to me believing that they need a mastectomy. Fortunately, for many of them I can say, 'No, you don't!"' she offered. "We are moving toward lessinvasive approaches, and this is great for our patients."

Breast conservation surgery with whole-breast radiation therapy (WBRT) is still the standard of care for

Presented by Amy Cyr, MD, Assistant Professor of Surgery at Washington University School of Medicine and a breast oncologic surgeon at the Siteman Cancer Center at Barnes-Jewish Hospital,

St. Louis, Missouri.

Dr. Cyr has disclosed that she has no financial interests, arrangement, affiliations, or commercial interests with the manufacturers of any products discussed in this article or their competitors.

Correspondence: Amy Cyr, MD, Siteman Cancer Center at BarnesJewish Hospital and Washington University School of Medicine,

4921 Parkview Avenue, St. Louis, MO 63110.

E-mail: cyra@wudosis.wustl.edu early-stage breast cancer, but many women are candidates for less aggressive treatment. This includes hypofractionated WBRT and accelerated partial breast irradiation (APBI), "but there are selection criteria that should be followed," Dr. Cyr cautioned.

Radiotherapy is also selectively omitted in women aged 70 years and older with favorable disease, she added.

\section{More Radiotherapy Options}

Hypofractionated WBRT is typically a 3-week course of 15 to 16 fractions given at higher doses per fraction than the standard 25-fraction WBRT, which is delivered over approximately 6 weeks. The selection of hypofractionated radiotherapy depends on the location of the partial mastectomy cavity, characteristics of the cancer, and the physics of treatment planning.

The American Society for Radiation Oncology (ASTRO) maintains that hypofractionation is an appropriate option for women over the age of 50 years treated with breast-conserving surgery with node-negative breast cancer and tumors less than $5 \mathrm{~cm}^{1}$; the NCCN Clinical Practice Guidelines in Oncology (NCCN Guidelines) for Breast Cancer promote this approach in appropriate patients.

"It is important to note that the ASTRO consensus panel did not indicate that patients who do not meet these criteria are not eligible. Other patients may also be good candidates, but data were lacking for a consensus," she added.

APBI involves an even shorter radiotherapy course, typically delivered twice a day for 5 days by balloon catheter, multicatheter brachytherapy, or single-catheter brachytherapy. The NCCN Guidelines now promote the use of short-course APBI with the caveat that follow-up of such patients has been limited and concerns have been raised about cosmesis. The guidelines 
also reference ASTRO's classification of appropriateness, per patient subset, as "suitable, cautionary, and unsuitable."

Reassuring data came from the final analysis of the American Society of Breast Surgeons MammoSite Breast Brachytherapy Registry Trial. ${ }^{2}$ After a median follow-up of 63 months, recurrence rates were "low and acceptable, and within the range of WBRT results," Dr. Cyr noted.

At 5 years, the ipsilateral recurrence rate was 3.7\% for invasive cases and $4.3 \%$ for ductal carcinoma in situ (DCIS) cases. Larger tumors, estrogen receptor-negative status, and "cautionary" or "unsuitable" category were prognostic indicators for recurrence.

Cosmetic results were comparable with those achieved with WBRT, though anecdotally, APBI may be associated with more fat necrosis ("lumps") at the lumpectomy site, which bothers some women, according to Dr. Cyr.

Given these caveats, she indicated, "There are huge benefits with APBI" in terms of access, convenience, good cosmesis, and reduced risk of radiation-related side effects.

Cosmetic results are also excellent with multiple interstitial catheter brachytherapy, but this has been replaced in a growing number of centers by the StrutAdjusted Volume Implant, single-catheter APBI modality. This modality allows for more individualized, flexible delivery of radiation and appears to result in less fat necrosis; however, formal data on these and oncologic outcomes are lacking. In a study presented at the ASTRO 2014 Annual Meeting, after 39 months median follow-up, the ipsilateral breast recurrence rate was $2.4 \%$ for invasive cancers and $4 \%$ for DCIS. ${ }^{3}$

\section{Advances in Molecular Profiling}

NCCN Guidelines support the use of Oncotype DX for determining which patients with node-negative, estrogen receptor-positive, early-stage breast cancer warrant chemotherapy. It also appears to have value in nodepositive disease, based on the TransATAC trial, ${ }^{4}$ which is reflected in a footnote added to the guidelines.

Other molecular profiling tools-MammaPrint, based on a 70-gene panel, and Prosigna, based on the PAM50 gene profile-may also be useful but are not recommended in the NCCN Guidelines.

None of these tools guide clinicians in treating patients believed to be at intermediate risk of recurrence, or those whose results are discordant with Adjuvant Online!. The MINDACT trial is expected to be informative on this issue, because it will test the value of chemotherapy in this challenging group.

"Research is evaluating these molecular profiling tests in different scenarios, to determine how best to use them," Dr. Cyr said. "We hope to be at a point soon where we can stratify patients with more precision as to who needs chemotherapy."

\section{Oncoplastics, Tumor Localization, and Radiofrequency Ablation}

Oncoplastics is a surgical strategy that combines the best oncologic methods with aesthetic techniques. The aim is to improve cosmetic results without compromising clinical outcomes. Involving both an oncologic surgeon and plastic surgeon, oncoplastics can incorporate breast reduction, tissue advancement flaps, incision placement, and skin removal. It offers improved cosmesis after partial mastectomy as it can correct for very large breasts and for the removal of substantial breast volume. It is also a very good method for correcting asymmetry.

Radioactive seed localization as an alternative to needle localization of the tumor is another surgical advance. This method helps surgeons "hone in" on the tumor, she said, "to make the incision where we want and resect a smaller volume of tissue."

Ablative therapies are also being evaluated, but none are yet approved. In a recent review of published studies of ultrasound-guided radiofrequency ablation (RFA), Grotenhuis et $\mathrm{al}^{5}$ found rates of complete $\mathrm{ab}$ lation in more than $90 \%$ of patients. However, they concluded that practical considerations existed that could be obstacles to RFA as treatment for earlystage breast cancer. Similarly, cryoablation is being evaluated, with high tumor ablation rates (94\%) in tumors less than $1 \mathrm{~cm}$, according to ACOSOG Z1072. ${ }^{6}$

\section{Avoiding Axillary Dissection}

Removal of lymph nodes can provide prognostic information, guide treatment decisions, and serve as tumor debulking. The question is whether this alters locoregional recurrence, distant recurrence, or survival.

"We still need lymph node staging information, at least for making decisions about adjuvant treat- 


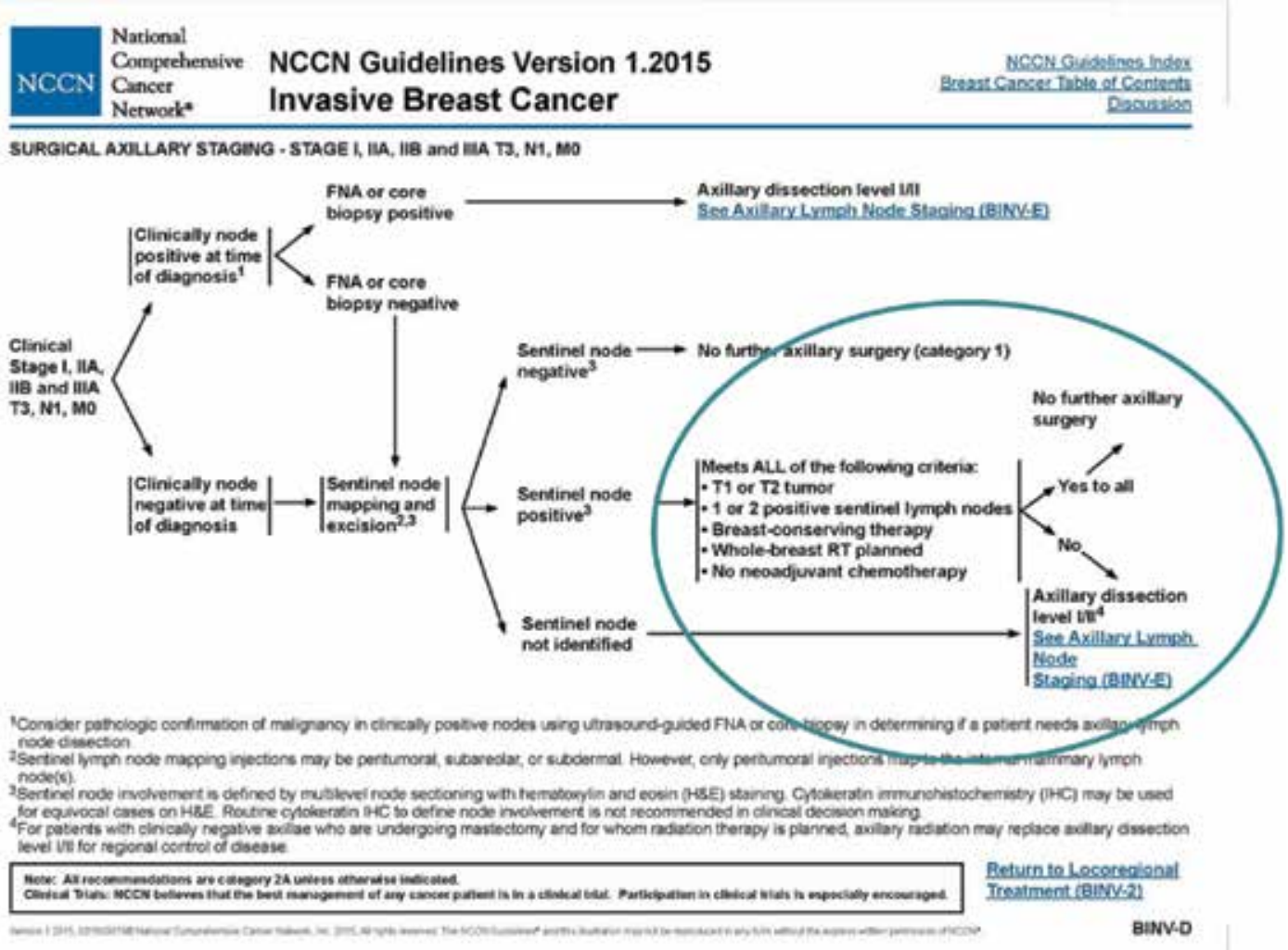

Figure1 NCCN Guidelines for Breast Cancer recommend no further axillary staging for a specific subset of patients.

ment, but we are not treating cancer by removing lymph nodes," Dr. Cyr indicated.

Decisions regarding postmastectomy radiation and axillary radiation may depend on the number of involved nodes, but tumor size, margin status, lymphovascular invasion, and extranodal extension also influence this decision, she added.

A practice-changing finding came from the ACOSOG Z0011 trial, which showed equivalent disease control for sentinel node biopsy alone and axillary dissection in a subset of patients with positive sentinel nodes, small tumors, and no clinically palpable nodes. ${ }^{7}$ This finding is now reflected in the NCCN Guidelines, which promote the avoidance of axillary dissection in this strict subset of patients (Figure 1).

The movement toward less-invasive axillary staging continues, using axillary ultrasound as a tool to evaluate the nodes for their size and shape. The Washington University experience has shown a negative predictive value of $84 \%$ for detecting micrometastatic or macrometastatic lymph node involvement and $89 \%$ for detecting macrometastatic
(> $2 \mathrm{~mm}$ ) disease. Further studies of this approach are underway in the United States and Europe.

\section{References}

1. Smith BD, Bentzen SM, Correa CR, et al. Fractionation for whole breast irradiation: an American Society for Radiation Oncology (ASTRO) evidence-based guideline. Int J Radiat Oncol Biol Phys 2011;81:59-68.

2. Shah C, Badiyan S, Ben Wilkinson J, et al. Treatment efficacy with accelerated partial breast irradiation (APBI): final analysis of the American Society of Breast Surgeons MammoSite breast brachytherapy registry trial. Ann Surg Oncol 2013;20:3279-3285.

3. Strasser J, Koprowski CD, Kuske R, et al. Outcomes for APBI with strutbased brachytherapy: 596 patients with 39-month median follow-up [abstract]. Int J Radiat Oncol Biol Phys 2014;90(Suppl):S271. Abstract 2134.

4. Dowsett M, Cuzick J, Wale C, et al. Prediction of risk of distant recurrence using the 21-gene recurrence score in node-negative and node-positive postmenopausal patients with breast cancer treated with anastrozole or tamoxifen: a TransATAC study. J Clin Oncol 2010;28:1829-1834.

5. Grotenhuis BA, Vrijland WW, Klem TM, et al. Radiofrequency ablation for early-stage breast cancer: treatment outcomes and practical considerations. Eur J Surg Oncol 2013;39:1317-1324.

6. Simmons R, Ballman K, Cox C, et al. A phase II trial exploring the success of cryoablation therapy in the treatment of invasive breast carcinoma. Results from ACOSOG (Alliance) Z1072 [abstract]. Ann Surg Oncol 2014;21(Suppl):S6

7. Giuliano AE, Hunt KK, Ballman KV, et al. Axillary dissection vs no axillary dissection in women with invasive breast cancer and sentinel node metastasis: a randomized clinical trial. JAMA 2011;305:569-575. 